\title{
Study of iterations in the design process of a product for automotive industry
}

\author{
Daniel-Constantin Anghel ${ }^{1, *}$, Nadia Belu ${ }^{1}$, Laurentiu-Mihai Ionescu ${ }^{2}$, and Nicoleta \\ Rachieru $^{1}$ \\ ${ }^{1}$ University of Pitesti, Department of Manufacturing and Industrial Management, Str. Tg. Din Vale, \\ nr. 1, Pitesti, Romania Romania \\ 2 University of Pitesti, Department of Electronics, Computers, Communications and Electrical \\ Engineering, Str. Tg. Din Vale, nr. 1, Pitesti, Romania
}

\begin{abstract}
This paper present an experiment realized in order to observe the iterations in a design process. The main objective of this work is to study the iterations during the design process using a laboratory experiment, in order to understand how and why iterations occur. The different forms of iterations as they occur in practice are identified. This study will help us in the classification of iterations in order to distinguish useful iterations from undesirable ones. The results of the study might be used to improve the manner of working in the field of engineering design.
\end{abstract}

\section{Introduction}

In this paper, a laboratory experimental was used to make observations about the iterations in a design process. Iteration is a fact of life in any project. The larger, more novel and more interconnected a project, the more of an issue it can be [1,2]. Iteration can be defined to be the repetition of design tasks due to the arrival or discovery of new information [3].

For the industries, engineering design is a source of competitive advantage. One of the key factor for the success for a corporation is the reducing the development time of the product development process.

For product developers, it is very important to understand iterations, so you can manage them through the design process.

But, the design process is a complex and dynamic one. In order to understand the design process, the experts have used a big number of methods and models. Most models are complementary each to other, the approaches used vary depending on the context, on the vision of experts and on the scope. Unfortunately, many of the models are developed based on intuition and practice.

The experiment presented in paper aims at observing and understanding iterations production, the effects of these iterations, how they can be anticipated etc.

This paper is organised as follows: a literature review of design process models integrating the iteration aspect of engineering design is presented. After this literature review, the research method used in this study and the experiment are presented. The next section

\footnotetext{
*Corresponding author: daniel.anghel@upit.ro
} 
presents the experimental data and observations, and their analysis. The conclusion of the paper present a summary of our work and a presentation of future developments.

\section{Literature review}

A way of understanding and acting on the design process is through its modelling. The work of developing a model provides a better understanding of the functional and behavioural characteristics of the design process. The use of the model makes it possible to define, test and improve strategies for the acquisition and engagement of design activities. The literature offers us a wide range of models. These models can be classified into two main families: prescriptive models and descriptive models [4]. Most of the models encountered and which can be used to study iterations are prescriptive models.

Smith and Eppinger [3] propose a model for the description of sequential processes with the presence of iterations. The model is used to find an initial order of activities leading to a minimal duration of the design process. Due to the assumptions adopted concerning the duration of the activities and the probabilities of questioning which are considered as constant and previously known.

The model developed by Krishnan [5] is used to manage the paralleling of two design activities. Downstream activity starts with partial information from upstream uncompleted activity. This can lead to poor premature design choices leading to loss of quality or causing long and costly iterations. The authors identified two characteristics of design process activities, called sensitivity and evolution, that allow us to choose the optimum degree of overlap to be adopted by determining information that can be frozen early in the design process and which information is to be used in a design process. Preliminary form. The limitations of this model are that it considers only the problem of overlapping only two activities. Moreover, it does not take into account the feedback from the downstream activity to the upstream activity and only downstream activity is reiterated in the process.

Yassine [6] also developed a mathematical model of two design activities that can be sequential, parallel, or partially overlapping. The duration of the iterations and their number are represented by random variables with a known distribution. The model calculates the total duration of the process.

Numerous research papers have approached the problem of iterations in the design process from close and distant. Indeed, these contribute greatly to the extension of development times. Osborne [7] reports that iterations account for between $13 \%$ and $70 \%$ of the total development time for Intel's semiconductor design activities. It also reports that the variation in development time is mainly due to iterations.

In their work, Pahl and Beitz describe the design process as a succession of phases [8]. They define iterations as the process by which a solution is approached step by step. They take place between the different phases and often within each phase. In this case, the iterations make it possible to refine a design solution. This procedure can be assimilated to that used in mathematics for solving an equation or a system of equations starting from an initial solution used to calculate another more precise solution which will in turn be used until convergence Towards an acceptable solution. This type of iteration is often encountered in interdependent design activities. For example, in order to carry out the activity $\mathrm{C}$, it is necessary to know the value of the parameter y provided by the activity $\mathrm{B}$. To calculate this parameter, the activity $\mathrm{B}$ in turn needs the value of the parameter $\mathrm{x}$ supplied by the activity $\mathrm{A}$, but this needs the result of the activity $\mathrm{C}$ represented by the parameter $\mathrm{z}$. In this case, an iterative process is required to compute all the parameters. 


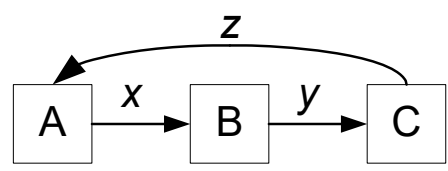

Fig. 1. A design iteration.

As a brief conclusion, in the literature, the iteration:

- $\quad$ is needed to solve complex problems [9];

- $\quad$ may help to deal with a changing context [9];

- may be reduced by a focus on central information-consuming/generating nodes [2];

- $\quad$ is strongly influenced by small changes in task time if close to capacity [10].

\section{Design Experiment}

The activity of design is a social one. In order to understand all the aspects of the design process, it is useful to use experimentations in order to observe how the designers interact, and how to process progress. Design could be accomplished in different situations: by an individual designer, or by a design team or several teams. The members of a design teams could work synchronously or asynchronously; they also could be geographically distributed [11].

The main objective of observations is to understanding cognition, creativity and innovation in the design process.

The role of our study is to observe iterations in a design activity performed by a multidisciplinary team. The observational techniques were used to record the design process. The records were used to make different study of the activity of participants.

The experiment described in this paper was developed at the University of Pitesti. The theme of the experiment was to design a roof lighting system for an SUV for intervention in situations of disasters or for the frontier service. The system must be able to adapt to all the atmospheric condition: clear sky, snowing, raining, fog, smog, pollution.

The experimental subjects were $4 \mathrm{PhD}$, three from the Department of Manufacturing and Industrial Management and one from Department of Electronics, Computers, Communications and Electrical Engineering. Each participant was given a distinct role. There were roles for a mechanical designer (Catia V5), an electrical and programmer engineer (Arduino), a risk analyst, and a mechanical specialist. The design team was led by a leader.

The experiment lasted for two weeks and there were 4 design sessions. All the sessions were recorded in order to fully capture the activity of design. In addition to these sessions, the designers performed their own tasks individually and asynchronously, but they could change information with others.
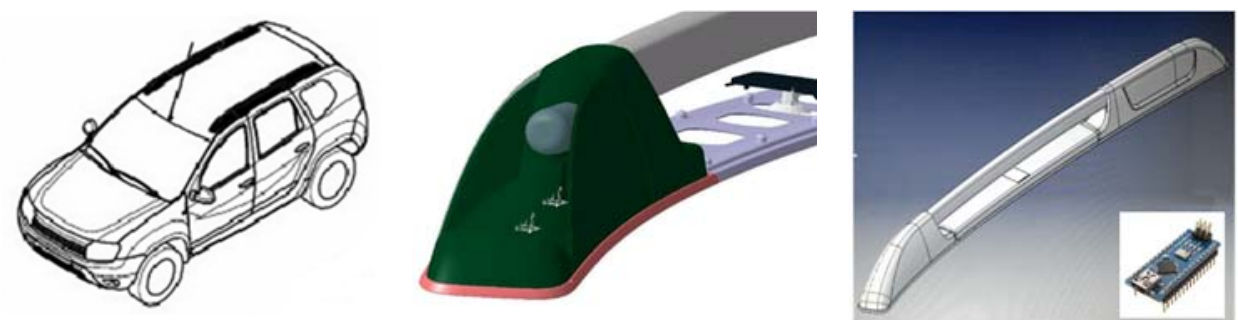

Fig. 2. The product - a roof lighting system. 


\section{Observations and results}

In this paper, it is presented the third working session, which corresponds to the maturity of the product "design embodiment." In this session, they were identified a great number of iterations.

The design tasks performed by the designers were established and then were identified all the iterations based on the links between the tasks, by use of DSM method, figure 3 .

\begin{tabular}{|c|r|r|r|r|r|r|r|r|r|r|r|r|r|r|r|l|}
\hline Task Name & 1 & 2 & 3 & 4 & 5 & 6 & 7 & 8 & 9 & 10 & 11 & 12 & 13 & 14 & 15 & 16 \\
\hline 1 & & & & & & & & & & & & & & & & \\
\hline 2 & 1 & 1 & & & & & & & & & & & 1 & \\
\hline 3 & 1 & & & & 1 & & & & & & & & & & \\
\hline 4 & & 1 & 1 & & & & & & & & & & & & \\
\hline 5 & & & 1 & 1 & & & & & & & & & & & \\
\hline 6 & & & & 1 & & & & & 1 & & & & & & \\
\hline 7 & & 1 & & 1 & & & & & & & & & & 1 & & \\
\hline 8 & & & & 1 & & & & & 1 & & & & & & & \\
\hline 9 & & & 1 & 1 & & & & 1 & & 1 & & & & & & 1 \\
\hline 10 & & & & & & & & & 1 & & & & & & 1 \\
\hline 11 & & 1 & & 1 & & 1 & & & 1 & & & & & & & \\
\hline 12 & & & & & & & & & & & 1 & & & & \\
\hline 13 & & & & 1 & & 1 & & & 1 & & & & & & \\
\hline 14 & & 1 & & & & & & & & & & 1 & & & \\
\hline 15 & & 1 & & & & & & & & & & 1 & & 1 & \\
\hline 16 & & & & & & & & & & & & & & 1 \\
\hline
\end{tabular}

Legend: Presentation of the problem; Analysis of solution for the roof bar; Analysis the location for the projectors; Choice of type of the projectors; Study of mechanical link projectors - bar; Electrical connection projectors - the command module; Detailing solutions for the fixture elements; Analysis of the command for the projectors; Solution development of the platform for analysis of atmospheric conditions; Developing the software for the platform; The risk analysis; The choice of materials; Evaluating the product in terms of ambient environmental conditions; Detailing solution for the roof bar; Rapid prototyping of the roof bar; Testing the solution for the system.

Fig. 3. The DSM representation of the third working session of design.

Design Structure Matrix (DSM) based models $[3,6]$ have been used to represent the iterative structure of engineering design. DSM uses a matrix representation of the design process. The DSM matrix is square with one task by column and by row. Information flows between tasks are indicated in the off-diagonal elements of the matrix. Two types of information flows are distinguished: feed forward (lower diagonal elements) and iterations (upper diagonal elements). With this representation, cyclic information flows are easily captured and the need for iterations is identified.

\subsection{The sources of the iterations}

After the analysis, we identified three main sources of iterations:

The change of objectives. During the design process, some initial data or solutions already proposed can be changed at any time for different reasons, leading to a repetition of a number of design tasks. 
Interdependence between the tasks. These are mutually dependent tasks, in this case several iterations are necessary to arrive at an acceptable solution. In this case, the total delay of the design process often depends on the initial scheduling of the interrelated design tasks.

Design errors. These errors are all the more important as the design process is more and more complex and involves more and more people, especially in the context of simultaneous engineering, where several different trades are taken together.

\subsection{The typology of iterations}

The iterations can be classified according to a multi-dimensional typology. We propose a typology with four classification criteria.

Voluntary / involuntary iterations. Voluntary iterations are due to interdependent activities and sometimes changes in objectives. Involuntary iterations are mainly due to design errors.

This classification makes it possible to better predict the actions to be taken to reduce the development time of the product. The reduction of iterative processes can be achieved by better structuring the activities of the design process: decoupling interdependent activities, better scheduling of design activities, reduction of coordination time between design teams, and so on. However, the reduction of iterative processes can increase the risk of failure of the solutions developed and must therefore be considered with caution.

Short/long iterations. An iteration can be considered short or long. This classification makes it possible to evaluate the impact of iterations on the time and/or the overall cost of the activities involved. This evaluation requires a form of quantification linked to the attributes of the activities (duration, cost) or the number of iterated activities. In this case, we must study the compromise between several short iterations and a limited number of long iterations.

Positive/negative iterations. Some iterations produce value, but not all of them. The iteration that can be eliminated without loss of value is considered a negative iteration. This classification makes it possible to specify how to handle the iterations (elimination, better control, reduction, etc.).

Fast/slow iterations. In this case, it is the speed at which a design task or part of a task is repeated to be in conformity with others or to correct errors.

In figure 4 the iterations are classified according to their sources.

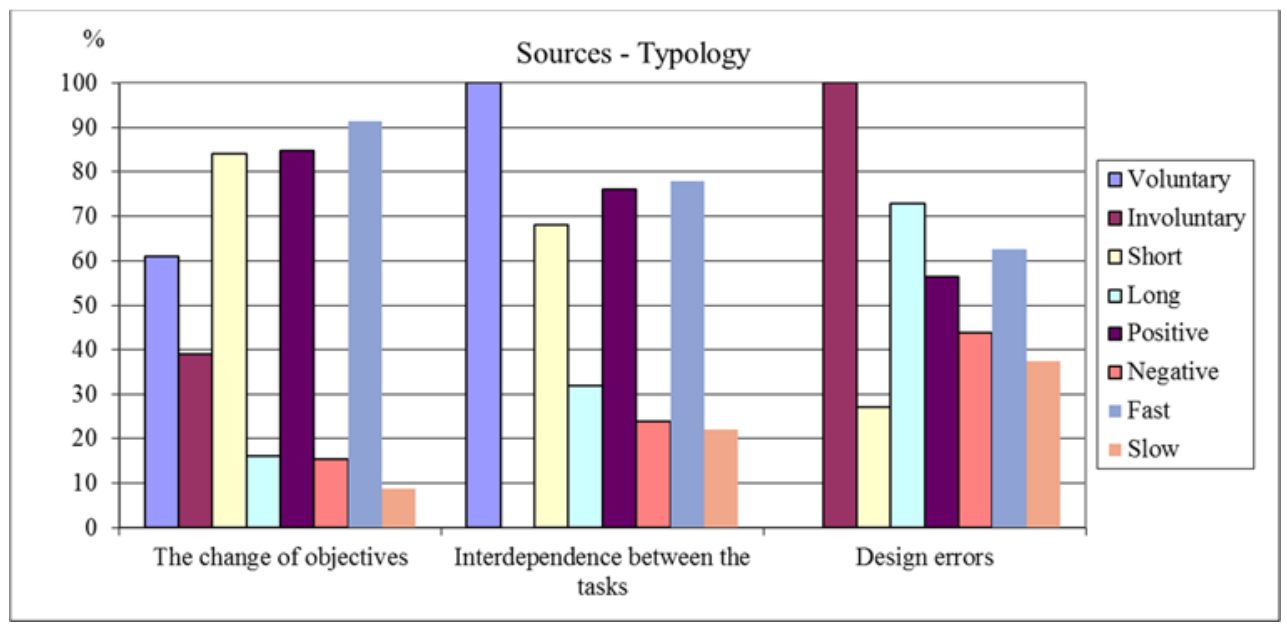

Fig. 4. Classification of iterations according to sources. 
Table 1. Sources/typology of iteration.

\begin{tabular}{|c|c|c|c|}
\hline $\begin{array}{c}\text { Sources/Typolog } \\
\mathrm{y}\end{array}$ & $\begin{array}{c}\text { The change of } \\
\text { objectives }\end{array}$ & $\begin{array}{c}\text { Interdependence between the } \\
\text { tasks }\end{array}$ & $\begin{array}{c}\text { Design } \\
\text { errors }\end{array}$ \\
\hline Voluntary & 61 & 100 & 0 \\
\hline Involuntary & 39 & 0 & 100 \\
\hline Short & 84 & 68,2 & 27,1 \\
\hline Long & 16 & 31,8 & 72,9 \\
\hline Positive & 84,7 & 76,2 & 56,4 \\
\hline Negative & 15,3 & 23,8 & 43,6 \\
\hline Fast & 91,3 & 77,9 & 62,6 \\
\hline Slow & 8,7 & 22,1 & 37,4 \\
\hline
\end{tabular}

\section{Conclusions}

Experiments provide data needed to understand the design process. In this study, we presented an iterations analysis on a design experiment.

Regarding changes of objectives, the iterations voluntary is more numerous because it is needed to explore the space of solutions in order to meet the new requirements of the design. Thus, a large number of short iterations, positive, is engaged to improve the chosen solution. A short iterations character makes iterative process to be done quickly.

It is very interesting that in the case of interdependence between the tasks, the involuntary iterations do not occur, which seems natural in theoretically. All these iterations are entered voluntarily, aiming to us "closer" to the final stage of the product.

In terms of design errors, it generates long iterations, affecting the duration of the design process. Unfortunately, these sources of iterations cannot be predicted, their appearance being made randomly.

This type of analysis of a design experience aids the project manager to build and distribute design teams, to optimize the design of a product in the new context of globalisation.

\section{References}

1. J. Mihm, C. Loch, A. Huchzermeier, Manage. Sci. 46(6), 733 (2003)

2. D. Braha, Y. Bar-Yam, Manage. Sci. 53(7), 1127 (2007)

3. R. P. Smith, S. D. Eppinger, Manage. Sci. 43, 1104 (1997)

4. S. Finger, J.R. Dixon, Research in Eng. Design. 1, 57 (1989)

5. V. Krishnan, S.D. Eppinger, Journal of Mech. Design 117, 491 (1995)

6. A. Yassine, K. Chelst, , D. Falkenburg, IEEE Trans. on Eng. Manage. 46, 2, 144 (1999)

7. S.M. Osborne, (M.I.T. 1992)

8. G. Pahl, W. Beitz (Springer-Verlag, 1997)

9. R.D. Moen, C.L. Norman, Qual. Prog. 43(11), 22 (2010)

10. B.A. Huberman, D.W. Wilkinson, Comput. Math. Organ. Theory. 11, 307 (2007)

11. T. Boudouh, D-C. Anghel, O. Garro, Advances in Design, 377 (Springer London, 2006) 